\section{Research Square}

Preprints are preliminary reports that have not undergone peer review.

They should not be considered conclusive, used to inform clinical practice, or referenced by the media as validated information.

\title{
Preoperative fibrinogen-to-prealbumin ratio and neutrophil lymphocyte ratio/prealbumin ratio versus platelet distribution width-to-platelet count ratio as a prognostic predictor for bladder cancer
}

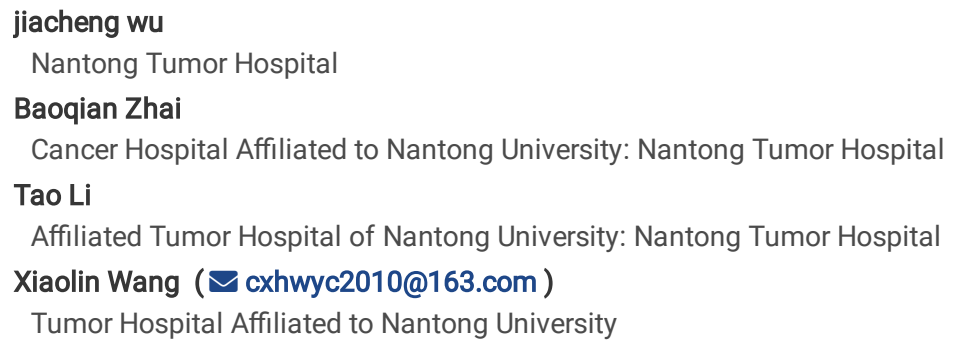




\section{Abstract \\ Background}

Evidence indicates that preoperative fibrinogen/prealbumin (FPR), neutrophil lymphocyte ratio/prealbumin ratio (NLR/PA) and platelet distribution width-toplatelet count ratio (PDW/PLT) possess prognostic potential in numerous malignancies. However, their roles in bladder cancer remain unclear. In this study, we investigated the association between FPR, NLR/PA versus PDW/PLT and the prognosis in bladder cancer patients.

\section{Methods}

The clinical data of 147 patients with bladder cancer treated in Nantong cancer hospital from January 2009 to August 2014 were retrospectively analyzed. According to ROC curve, the optimal critical value of FPR, NLR/PA and PDW/PLT were $0.1084,0.1045$ and 0.1210 respectively. The patients were followed up for 5 years to observe the survival of the patients, and the clinicopathological data were statistically analyzed. Cox regression analysis was used for univariate and multivariate analysis. Finally, on this basis, the nomogram is constructed for internal verification.

\section{Results}

All patients were followed up for 5 years. A total of 102 patients survived with a survival rate of $69.4 \%, 45$ patients died with a mortality rate of $30.6 \%$. Further stratified analysis showed that the group with low FPR, low NLR/PA and low PDW/PLT had the best prognosis, while the group with high FPR, high NLR/PA and high PDW/PLT had the worst prognosis. Cox multivariate analysis showed that preoperative FPR, NLR/PA and PDW/PLT were independent risk factors for tumor progression $(p=0.007, p=0.013, p=0.000)$. The decrease of FPR, NLR/PA and PDW/PLT can significantly prolong OS and PFS in patients with bladder cancer. In internal validation, the c-index of the nomogram was 0.8140 (95\% Cl: $0.7577-0.8703$ ).

\section{Conclusions}

Preoperative FPR and NLR/PA versus PDW/PLT can be an independent prognostic factor in bladder cancer patients and are associated with clinicopathological characteristics. They have a specific value in assessing the prognosis of bladder cancer patients.

\section{Introduction}

Bladder cancer is the most common malignant tumor of urinary system. According to the data of American Cancer Society, the number of new cases and deaths of bladder cancer in 2019 are 80470 and 17670, respectively [1]. In recent years, the incidence rate and mortality rate of bladder cancer in China also showed an upward trend [2]. In the management of bladder cancer patients, postoperative tumor recurrence and progression are important clinical endpoints [3,4]. Traditional TNM staging system can predict the prognosis of bladder cancer. However, in patients with similar TNM stages, the clinical outcomes were significantly different [5]. Although molecular diagnostic tests can be used in Europe and the United States to obtain more prognostic information and help guide clinical treatment, they cannot be used in routine clinical practice due to their high cost. Therefore, it is very important to identify low-cost biomarkers that can be easily obtained by routine blood cell count.

Nutrition and inflammation play an important role in the progression and prognosis of cancer. At present, many studies have reported related inflammatory markers to predict the prognosis of bladder cancer patients, such as platelet lymphocyte ratio (PLR) [6], neutrophil lymphocyte ratio (NLR) [7, 8], lymphocyte monocyte ratio (LMR) $[9,10]$. Recent studies have shown that fibrinogen and its derived peptides play a pro-inflammatory role in solid cancer [11]. In addition, emerging evidence suggests that elevated fibrinogen is associated with poor OS and tumor free survival [12]. In addition to fibrinogen, prealbumin (PA) is a common nutritional indicator, and low preoperative PA level is an independent negative prognostic factor for cancer-specific survival [13]. Current studies also have found that platelet related markers, such as the ratio of platelet to lymphocyte, are more significant prognostic factors in patients with bladder cancer [14].

Recently, it has been reported that three new predictive indexes, one called preoperative fibrinogen ratio to prealbumin ratio (FPR), is related to the prognosis of patients with liver cancer [15], one is neutrophil lymphocyte ratio to prealbumin ratio (NLR/PA), which is related to the prognosis of patients with esophageal cancer [16]. Another one is that the platelet distribution width-to-platelet count ratio (PDW/PLT) has a significant prognosis in breast cancer patients [17]. However, up to now, there is no report on the relationship between FPR, NLR/PA and PDW/PLT and the prognosis of bladder cancer patients. Most of the current studies predict the prognosis of tumor through a single predictive index, which has certain limitations in accuracy and effectiveness. Therefore, the purpose of this study is to evaluate the prognosis of patients with bladder cancer by combining preoperative FPR, NLR/PA and PDW/PLT.

\section{Methods}

Clinical data

The clinical data of bladder cancer patients admitted to the urology department of Nantong tumor hospital from January 2009 to August 2014 were selected. Inclusion criteria: (I) all patients were diagnosed as bladder cancer for the first time and operated on; (II) no upper respiratory tract infection, urinary tract infection, fever and other factors affecting the results of blood routine examination within 1 month before operation; (III)the last blood routine 
examination before operation, calculated the ratio of fibrinogen to prealbumin, the neutrophil lymphocyte ratio to prealbumin and platelet distribution widthto-platelet count ratio; (IV)complete clinical, pathological and follow-up data. Exclusion criteria: (I) patients with positive pathological margin after operation; (II) patients with history of blood system, autoimmune diseases and other malignant tumors; (III)patients with chronic obstructive pulmonary disease, heart failure, coronary heart disease, high-risk hypertension and other factors affecting postoperative recovery; (IV)patients with long-term radiation exposure before operation and neoadjuvant chemotherapy; (V)patients with serious complications during perioperative period; (VI)Non urothelial tumor; (『)antibiotics, anticoagulants, hormones and other factors have an impact on the blood test results in the near future. 147 patients were included in the study, including 119 males and 28 females. The average age was $(63.0 \pm 11.5)$ years.

Research methods

Through the medical record management system of our hospital, we collected the last blood routine examination and the corresponding clinicopathological data. Neutrophils, lymphocytes, fibrinogen, prealbumin, platelet distribution width, and platelet count in blood routine were recorded, then FPR, NLR/PA and PDW/PLT were calculated. The ROC curves of FPR, NLR/PA and PDW/PLT were established to calculate the Youden index. The FPR value, NLR/PA value and PDW/PLT value corresponding to the maximum value were selected as the best cut-off point. The patients were divided into high FPR group, low FPR group, high NLR/PA group, low NLR/PA group, high PDW/PLT group and low PDW/PLT group. Postoperative OS and PFS were used as survival analysis indexes, and the clinicopathological data and survival differences between the two groups were compared. At the same time, univariate and multivariate prognostic analysis was performed.

Follow-up

The follow-up methods were outpatient review, re-admission medical records and telephone follow-up. The patients were followed up once every three months in the first year, once every six months in the second year, and once a year after two years. All patients were followed up to August 1,2019 . Follow up items: Cystoscopy, abdominal and pelvic CT or color Doppler ultrasound, chest X-ray, tumor markers, urine routine, liver and kidney function and so on (additional examination depending on the patient's condition). OUTCOME MEASURES: PFS was defined as the indication of disease progression from the beginning of treatment to any follow-up items. The data of survival at the end of follow-up and loss of follow-up were used as the final cut-off time for statistical analysis.

\section{Statistical analysis}

SPSS 22.0 statistical software was used to process the data. ROC curve was used to calculate the best cut-off value. The measurement data were expressed by $\mathrm{X} \pm \mathrm{s}$ and compared by $\mathrm{t}$ test; the count data were expressed by \% and compared by chi-square test or Fisher's exact probability test. Kaplan Meier method and log rank test were used to test the effect on OS and PFS. Factors with statistical significance in univariate analysis were included in multivariate Cox proportional hazards regression model. Finally, on this basis, R language is used to draw the nomogram and build the prediction model. The difference was statistically significant $(p<0.05)$.

\section{Results}

Relationship between FPR, NLR/PA, PDW/PLT and tumor progression

The ROC curve of the relationship between FPR, NLR/PA, and PDW/PLT and tumor progression is shown in Fig. 1. The best cut-off value of FPR determined by ROC curve was 0.1084 . According to whether the preoperative FPR was greater than 0.1084 , the patients were divided into low FPR group ( $\mathrm{n}=85$ ) and high FPR group $(n=62)$. The optimal cut-off value of NLR/PA was 0.1046 . According to whether the preoperative NLR/PA was greater than 0.1046 , the patients were divided into low NLR/PA group $(n=82)$ and high NLR/PA group $(n=65)$. The optimal critical value of PDW/PLT was 0.1210 . The patients were divided into low PDW/PLT group $(n=123)$ and high PDW/PLT group $(n=24)$ according to whether the preoperative PDW/PLT was greater than 0.1210 .

Comparison of OS and PFS between the two groups

The tumor progression rates of low FPR group and high FPR group were $30.6 \%(26 / 85)$ and $61.3 \%$ (38/62), and the median PFS was 67.0 months and 55.0 months. The OS and PFS of low FPR group were significantly higher than that of high FPR group $(\mathrm{p}<0.05)$, as shown in Fig. 2 and Fig. 3 . The tumor progression rates of low NLR/PA group and high NLR/PA group were $32.9 \%$ (27/82) and 47.7\% (31/65), and the median PFS was 72.5 months and 55 months, respectively. The OS and PFS of low NLR/PA group were significantly higher than that of high NLR/PA group ( $p<0.05)$, as shown in Fig. 4 and Fig. 5. The tumor progression rates of low PDW/PLT group and high PDW/PLT group were 29.2\% (36/123) and 51.2\% (13/24), and the median PFS were 69.8 months and 59 months, respectively, as shown in Fig. 6 and Fig. 7.

Comparison of OS and PFS of eight groups after stratified analysis

Further stratified analysis showed that the group with low FPR, low NLR/PA, low PDW/PLT had the best prognosis, while the group with high FPR, high NLR/PA and high PDW/PLT had the worst prognosis, as shown in Fig. 8 and Fig. 9.

Patients and tumor characteristics

There were significant differences in TNM stage, grade and history of hypertension between low FPR group and high FPR group ( $<<0.05$ ). The number of patients with history of hypertension in low NLR/PA group was significantly less than that in high NLR/PA group $(p=0.017)$. Low PDW/PLT group and high 
PDW/PLT group also had significant difference in grade $(p<0.05)$, as shown in Table 1.

Table 1

The relationship between preoperative FPR, NLR/PA and PDW/PLT and clinicopathological data

\begin{tabular}{|c|c|c|c|c|c|c|c|c|c|c|}
\hline \multirow[t]{2}{*}{ Variable } & Total & $\begin{array}{l}\text { FPR } \leq \\
0.1084\end{array}$ & FPR囚0.1084 & \multirow[t]{2}{*}{$p$} & \multicolumn{2}{|l|}{$\begin{array}{l}\text { NLR/PA } \\
\leq \\
0.1045\end{array}$} & \multirow[t]{2}{*}{$p$} & $\begin{array}{l}\text { PDW/PLT } \\
\leq 0.1210\end{array}$ & PDW/PLT冈0.1210 & \multirow[t]{2}{*}{$P$} \\
\hline & $n(\%)$ & $n(\%)$ & $n(\%)$ & & $n(\%)$ & $n(\%)$ & & $n(\%)$ & $\mathrm{n}(\%)$ & \\
\hline \multicolumn{11}{|l|}{ Age } \\
\hline$\leq 65$ & 74(50.3) & $42(56.8)$ & $32(43.2)$ & 0.792 & $44(59.5)$ & $30(40.5)$ & 0.366 & $37(50.0)$ & $37(50.0)$ & 0.298 \\
\hline 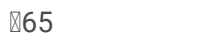 & 73(49.7) & $43(58.9)$ & $30(41.1)$ & & $38(52.1)$ & $35(47.9)$ & & $45(61.6)$ & 73(38.4) & \\
\hline \multicolumn{11}{|l|}{ Gender } \\
\hline male & $119(81.0)$ & 71(59.7) & $48(40.3)$ & 0.352 & $67(56.3)$ & $52(43.7)$ & 0.793 & $52(43.7)$ & $67(56.3)$ & 0.513 \\
\hline female & $28(19.0)$ & $14(50.0)$ & $14(50.0)$ & & 15(53.6) & $13(46.4)$ & & $22(78.6)$ & $6(21.4)$ & \\
\hline \multicolumn{11}{|l|}{ TNM stage } \\
\hline $\mathrm{T} 1$ & $91(61.9)$ & $62(68.1)$ & 29(31.9) & $0.001^{\star}$ & $52(57.1)$ & $39(42.9)$ & 0.672 & $41(45.1)$ & $50(54.9)$ & 0.142 \\
\hline $\mathrm{T} 2+\mathrm{T} 3$ & $56(38.1)$ & $23(41.1)$ & $33(58.9)$ & & $30(53.6)$ & $26(46.4)$ & & $40(71.4)$ & $16(28.6)$ & \\
\hline \multicolumn{11}{|l|}{ Grade } \\
\hline $\mathrm{G} 1+\mathrm{G} 2$ & $91(61.9)$ & 63(69.2) & $28(30.8)$ & $0.000 *$ & $51(56.0)$ & $40(44.0)$ & 0.935 & $39(42.9)$ & $52(57.1)$ & $0.003^{*}$ \\
\hline G3 & $56(38.1)$ & $22(39.3)$ & $34(60.7)$ & & $31(55.4)$ & $25(44.6)$ & & $27(48.2)$ & $29(51.8)$ & \\
\hline \multicolumn{11}{|l|}{$\begin{array}{l}\text { Lymph node } \\
\text { metastasis }\end{array}$} \\
\hline Yes & $7(4.8)$ & $2(28.6)$ & $5(71.4)$ & 0.108 & $2(28.6)$ & $5(71.4)$ & 0.137 & $5(71.4)$ & $2(28.6)$ & 0.098 \\
\hline No & $140(95.2)$ & 83(59.3) & $57(40.7)$ & & $80(57.1)$ & $60(42.9)$ & & $73(52.1)$ & $67(47.9)$ & \\
\hline \multicolumn{11}{|l|}{$\begin{array}{l}\text { Distant } \\
\text { metastasis }\end{array}$} \\
\hline Yes & $2(1.4)$ & $1(50.0)$ & $1(50.0)$ & 0.822 & $2(100.0)$ & $0(0.0)$ & 0.205 & $1(50.0)$ & $1(50.0)$ & 0.138 \\
\hline No & 145(98.6) & $84(57.9)$ & $61(42.1)$ & & $80(55.2)$ & $65(44.8)$ & & $91(62.8)$ & $54(37.2)$ & \\
\hline \multicolumn{11}{|l|}{$\begin{array}{l}\text { Operation } \\
\text { method }\end{array}$} \\
\hline $\begin{array}{l}\text { Radical } \\
\text { resection }\end{array}$ & $48(32.7)$ & $26(54.2)$ & $22(45.8)$ & 0.532 & $24(50.0)$ & $24(50.0)$ & 0.326 & $25(52.1)$ & $23(47.9)$ & 0.472 \\
\hline $\begin{array}{l}\text { Electrosurgical } \\
\text { resection }\end{array}$ & $99(67.3)$ & $59(59.6)$ & $40(40.4)$ & & $58(58.6)$ & $41(41.4)$ & & $51(51.5)$ & $48(48.5)$ & \\
\hline \multicolumn{11}{|l|}{$\begin{array}{l}\text { History of } \\
\text { hypertension }\end{array}$} \\
\hline Yes & $26(17.7)$ & 21(80.8) & $5(19.2)$ & $0.009 *$ & $20(76.9)$ & $6(23.1)$ & $0.017 *$ & 23(88.5) & $3(11.5)$ & 0.040 * \\
\hline No & $121(82.3)$ & $64(52.9)$ & $57(47.1)$ & & $62(51.2)$ & $59(48.8)$ & & $59(48.8)$ & $62(51.2)$ & \\
\hline \multicolumn{11}{|l|}{$\begin{array}{l}\text { History of } \\
\text { diabetes }\end{array}$} \\
\hline Yes & $6(4.1)$ & $2(33.3)$ & $4(66.7)$ & 0.215 & 2(33.3) & $4(66.7)$ & 0.258 & $3(50.0)$ & $3(50.0)$ & 0.256 \\
\hline no & $141(95.9)$ & 83(58.9) & $58(41.1)$ & & $80(56.7)$ & $61(43.3)$ & & $75(53.2)$ & $66(46.8)$ & \\
\hline \multicolumn{11}{|l|}{$\begin{array}{l}\text { Other } \\
\text { operation } \\
\text { history }\end{array}$} \\
\hline Yes & $41(27.9)$ & $24(58.5)$ & $17(41.5)$ & 0.913 & 19(46.3) & $22(53.7)$ & 0.152 & $29(70.7)$ & 12(29.3) & 0.141 \\
\hline No & $106(72.1)$ & $61(57.5)$ & $45(42.5)$ & & $63(59.4)$ & $43(40.6)$ & & $53(50.0)$ & $53(50.0)$ & \\
\hline
\end{tabular}

${ }^{\star} P<0.05$ was statistically significant. 
Univariate analysis results

Univariate analysis showed that TNM stage, grade, FPR, NLR/PA, PDW/PLT were the influencing factors of $0 S(p<0.05)$, as shown in Table 2. 
Table 2

Univariate analysis of clinical factors on OS

\begin{tabular}{|c|c|c|c|}
\hline Variable & HR & $(95 \% \mathrm{Cl})$ & $p$ \\
\hline \multicolumn{4}{|l|}{ Age } \\
\hline$\leq 65$ & 1.183 & $(0.654-2.143)$ & 0.578 \\
\hline$\varangle 65$ & 1 & & \\
\hline \multicolumn{4}{|l|}{ Gender } \\
\hline male & 0.719 & $(0.304-1.702)$ & 0.453 \\
\hline female & 1 & & \\
\hline \multicolumn{4}{|l|}{ TNM stage } \\
\hline T1 & 2.137 & $(1.183-3.861)$ & $0.012^{\star}$ \\
\hline $\mathrm{T} 2+\mathrm{T} 3$ & 1 & & \\
\hline \multicolumn{4}{|l|}{ Grade } \\
\hline $\mathrm{G} 1+\mathrm{G} 2$ & 3.457 & $(1.876-6.369)$ & $0.000 *$ \\
\hline G3 & 1 & & \\
\hline \multicolumn{4}{|l|}{ Lymph node metastasis } \\
\hline Yes & 0.475 & $(0.170-1.331)$ & 0.157 \\
\hline No & 1 & & \\
\hline \multicolumn{4}{|l|}{ Distant metastasis } \\
\hline Yes & 20.612 & $(0.001-641741.404)$ & 0.566 \\
\hline No & 1 & & \\
\hline \multicolumn{4}{|l|}{ Operation method } \\
\hline Radical resection & 0.708 & $(0.388-1.293)$ & 0.261 \\
\hline Electrosurgical resection & 1 & & \\
\hline \multicolumn{4}{|l|}{ History of hypertension } \\
\hline Yes & 1.244 & $(0.554-2.792)$ & 0.596 \\
\hline No & 1 & & \\
\hline \multicolumn{4}{|l|}{ History of diabetes } \\
\hline Yes & 0.481 & $(0.149-1.554)$ & 0.221 \\
\hline no & 1 & & \\
\hline \multicolumn{4}{|l|}{ Other operation history } \\
\hline Yes & 0.797 & $(0.422-1.504)$ & 0.483 \\
\hline No & 1 & & \\
\hline \multicolumn{4}{|l|}{ FPR } \\
\hline$\leq 0.1084$ & 4.362 & $(2.283-8.335)$ & $0.000 *$ \\
\hline$\bowtie 0.1084$ & 1 & & \\
\hline \multicolumn{4}{|l|}{ NLR/PA } \\
\hline$\leq 0.1045$ & 3.088 & $(1.660-5.744)$ & $0.000 *$ \\
\hline$\bowtie 0.1045$ & 1 & & \\
\hline \multicolumn{4}{|l|}{ PDW/PLT } \\
\hline$\leq 0.1210$ & 5.439 & $(2.929-10.100)$ & $0.000 *$ \\
\hline$凶 0.1210$ & 1 & & \\
\hline
\end{tabular}

$\star P<0.05$ was statistically significant. 
Multivariate analysis results

Cox multivariate analysis showed that preoperative FPR, NLR/PA and PDW/PLT were independent risk factors for tumor progression $(p=0.007, p=0.013, p$ $=0.000)$, and tumor grade also had a certain impact on tumor progression $(p=0.005)$, as shown in Table 3 .

Table 3

Cox regression analysis of prognostic factors in

\begin{tabular}{|c|c|c|c|}
\hline Variable & HR & $(95 \% \mathrm{Cl})$ & $p$ \\
\hline \multicolumn{4}{|l|}{ Grade } \\
\hline $\mathrm{G} 1+\mathrm{G} 2$ & 0.399 & $(0.210-0.758)$ & $0.005^{\star}$ \\
\hline G3 & 1 & & \\
\hline \multicolumn{4}{|l|}{ FPR } \\
\hline$\leq 0.1084$ & 0.379 & $(0.187-0.770)$ & $0.007^{*}$ \\
\hline$\llbracket 0.1084$ & 1 & & \\
\hline \multicolumn{4}{|l|}{ NLR/PA } \\
\hline$\leq 0.1045$ & 0.441 & $(0.230-0.844)$ & $0.013^{*}$ \\
\hline$\otimes 0.1045$ & 1 & & \\
\hline \multicolumn{4}{|l|}{ PDW/PLT } \\
\hline$\leq 0.1210$ & 0.169 & $(0.088-0.325)$ & $0.000 *$ \\
\hline$\varangle 0.1210$ & 1 & & \\
\hline
\end{tabular}

$\star P<0.05$ was statistically significant.

$\mathrm{R}$ language to draw nomogram and build prediction model

Based on the results of Cox regression analysis, the nomogram could accurately predict the 3-year and 5-year survival rates of patients with different grade, FPR, NLR/PA and PDW/PLT. Meanwhile, in the internal validation, the c-index of the nomogram is $0.8140(95 \% \mathrm{Cl} 0.7577-0.8703)$, so the prediction model has good prediction accuracy, as shown in Fig. 10.

\section{Discussion}

This study found that FPR, NLR/PA, PDW/PLT are associated with the survival and prognosis of patients, and are independent risk factors for the prognosis of bladder cancer. Our findings indicate the potential importance of combining clinicopathological features with FPR, NLR/PA and PDW/PLT to evaluate the prognosis of bladder cancer.

Fibrinogen is a multifunctional protein that affects many cellular processes in tumorigenesis and metastasis. Fibrinogen is one of the most common components of extracellular matrix, which connects to tumor cells. Fibrinogen generates proliferation signals by acting as a scaffold for binding growth factors such as FGF-2 and VEGF. Growth factor binding promotes cell adhesion, proliferation and migration during angiogenesis and tumor cell growth [18]. In addition, cancer cells can synthesize and secrete additional endogenous fibrinogen, high FIB can promote the synthesis of IL- 6 and change the nature of leukocyte infiltration, and stimulate T cells and B cells to promote chronic inflammatory response [19-21].

Immune cells play an important role in occurrence, development and metastasis of tumors. Neutrophils can interact with tumor cells and secrete cytokines and chemokines, thus promoting tumor proliferation, angiogenesis and metastasis [22]. It has been reported that neutrophils can secrete vascular endothelial growth factor (VEGF) into the circulation, and VEGF is essential for tumor angiogenesis, metastasis and drug resistance. On the other hand, the role of lymphocytes is mainly to inhibit the occurrence and development of tumor through tumor immune monitoring and tumor cell clearance. At the same time, neutrophils in the tumor microenvironment can also work with lymphocytes to reduce the anti-tumor effect of activated T cells [23, 24]. Therefore, increased NLR can represent neutropenia and lymphopenia, which reflects the imbalance of immune response.

Malnutrition often occurs in patients with malignant tumors. Serum prealbumin is a negative acute phase protein synthesized by the liver, which is often used to evaluate the nutritional status of patients with malignant tumors $[25,26]$. Serum prealbumin is a stable tetramer composed of four identical subunits synthesized by liver. Each subunit contains 127 amino acid residues with a relative molecular weight of 61000 . It is named after the migration position before albumin in electrophoresis. The content of prealbumin in normal people's serum is very little, and its half-life in vivo is only $1.9 \mathrm{D}$, which is involved in the transport and regulation of vitamin A and thyroxine. At the same time, prealbumin also has thymic activity, which can enhance the body's immunity by promoting lymphocyte maturation, and has potential anti-tumor effect [27-31].

Although there are many recent studies on the clinical significance of activated platelets in cancer, the range of available data is still limited by the type of malignant tumor and the clinical results studied. Platelets are rich in PDGF, transforming growth factor- $\beta$ and platelet-derived endothelial growth factor.

Page 7/19 
These platelet-derived growth factors are usually produced in large quantities by cancer cells and contribute to their development [32, 33]. Thrombocytosis is associated with reduced survival in patients with a variety of tumor types, including rectal cancer, lung cancer, renal cancer, gastric cancer, ovarian cancer, brain cancer, endometrial cancer, pancreatic cancer, and breast cancer. Elevated platelets promote cancer progression and metastasis by shielding circulating tumor cells from immune surveillance and killing [34]. For bladder cancer, the increased expression of platelet-derived endothelial growth factor is significantly correlated with the tumor progression of bladder cancer [35].

PDW is a more specific marker of platelet activation, because it will not increase due to platelet swelling, and it is also a method to measure platelet heterogeneity caused by megakaryocyte heterogeneity demarcation [36]. A high value of this index indicates the presence of both mature and immature cells in the circulation. This means that the increase of PDW may be accompanied by abnormal thrombosis [35] and/or the result of heterogeneous boundary of megakaryocytes [37]. The cause of poor prognosis in patients with high PDW/PLT is unclear. Inflammation may be the link between PDW/PLT and survival. There is a strong link between inflammation and cancer [37,38]. Various pro-inflammatory cytokines are up-regulated with the progress of tumor, and promote the maturation of heterologous megakaryocytes, leading to the production and release of immature platelets with various characteristics and sizes into the circulatory system to meet the growing demand of tumor [39]. However, further studies are needed to better understand the causes of poor prognosis of bladder cancer patients with high PDW/PLT.

In this study, we combined these three indicators to evaluate the prognosis of patients with bladder cancer, and the relationship between preoperative FPR, NLR/PA and PDW/PLT and the patients' OS and PFS. We can find that the prognosis of low FPR group is better than that of high FPR group, the prognosis of low NLR/PA group is better than that of high NLR/PA group, and the prognosis of low PDW/PLT group is better than that of high PDW/PLT group. Further stratified analysis showed that the group with low FPR, low NLR/PA and low PDW/PLT had the best prognosis, while the group with high FPR, high NLR/PA and high PDW/PLT had the worst prognosis. Cox regression analysis showed that TMN stage, grade, FPR, NLR/PA and PDW/PLT were statistically significant. At the same time, in order to avoid the influence of these factors, we included these five factors into the multivariate analysis. Finally, we found that the grade, FPR, NLR/PA and PDW/PLT are independent factors affecting the prognosis of patients with bladder cancer. That is, for patients with bladder cancer, the smaller the FPR value, the smaller the NLR/PA value, the smaller the PDW/PLT value, and the lower the grade, the longer the OS and PFS. At the same time, we found that at least 20 patients had at least one relapse, and 52 patients with NMIBC finally developed into MIBC. This is consistent with the characteristics of multicentric growth, easy recurrence and progression of bladder cancer, and also reflects the positive clinical significance of this study. Based on the results of Cox regression analysis, we drew a nomogram and carried out internal verification, so as to have a more accurate judgment on the prediction of 3-year and 5-year survival rate of bladder cancer patients, which also has a certain practical significance for the development of clinical treatment. In the internal validation, the c-index of the nomogram is $0.8140(95 \% \mathrm{Cl} 0.7577-0.8703)$, so the prediction model has good accuracy.

This study also has some limitations, including single center design and relatively small sample size. At the same time, this is a retrospective study, which may lead to bias in data selection and analysis. Despite these limitations, this study is still the first to reveal that elevated FPR, NLR/PA and PDW/PLT are predictors of poor prognosis in patients with bladder cancer.

\section{Conclusions}

Although there are some limitations in this study, the data clearly show that the increase of preoperative FPR, NLR/PA and PDW/PLT is an unfavorable prognostic factor for patients with bladder cancer. Further studies are needed to elucidate the exact mechanism of FPR, NLR/PA and PDW/PLT in bladder cancer.

\section{Declarations}

Acknowledgments

Declared none.

\section{Funding}

This work was supported financially by grants from the Nantong Science and technology plan fund (MS22019010), Youth fund of Nantong Health Commission (QA2020023), and Clinical medicine program of Nantong University (Youth Program - 2019LQ013, 2019LQ011).

\section{Availability of data and materials}

Authors can confirm all relevant data are included in the article and materials are available on request from the authors.

\section{Authors' contributions}

All authors made substantial contributions to conception and design, acquisition of data, or analysis and interpretation of data; took part in drafting the article or revising it critically for important intellectual content; agreed to submit to the current journal; gave final approval of the version to be published; and agree to be accountable for all aspects of the work.

\section{Ethics approval and consent to participate}


The study was approved by The Ethics Committee of the Tumor Hospital Affiliated to Nantong University (Nantong, China). Written informed consent was obtained from all individual participants included in the study. And this study was conducted in accordance with the Declaration of Helsinki.

\section{Consent for publication}

Not applicable.

\section{Competing interests}

The authors declare that they have no competing interests

\section{References}

1. Siegel RL, Miller KD, Jemal A. Cancer statistics, 2019. CA Cancer J Clin. 2019;69(1):7-34.

2. Li K, Lin T, Chinese Bladder Cancer C, Xue W, Mu X, Xu E, Yang X, Chen F, Li G, Ma L, et al. Current status of diagnosis and treatment of bladder cancer in China - Analyses of Chinese Bladder Cancer Consortium database. Asian J Urol. 2015;2(2):63-9.

3. Alfred Witjes J, Lebret T, Comperat EM, Cowan NC, De Santis M, Bruins HM, Hernandez V, Espinos EL, Dunn J, Rouanne M, et al. Updated 2016 EAU Guidelines on Muscle-invasive and Metastatic Bladder Cancer. Eur Urol. 2017;71(3):462-75.

4. Chang SS, Bochner BH, Chou R, Dreicer R, Kamat AM, Lerner SP, Lotan Y, Meeks JJ, Michalski JM, Morgan TM, et al. Treatment of Non-Metastatic Muscle-Invasive Bladder Cancer: AUA/ASCO/ASTRO/SUO Guideline. J Urol. 2017;198(3):552-9.

5. Mitra AP, Daneshmand S. Molecular Prognostication in Bladder Cancer. Cancer Treat Res. 2018;175:165-91.

6. Peng D, Gong YQ, Hao H, He ZS, Li XS, Zhang CJ, Zhou LQ. Preoperative Prognostic Nutritional Index is a Significant Predictor of Survival with Bladder Cancer after Radical Cystectomy: a retrospective study. BMC Cancer. 2017;17(1):391.

7. Tazeh NN, Canter DJ, Damodaran S, Rushmer T, Richards KA, Abel EJ, Jarrard DF, Downs TM. Neutrophil to Lymphocyte Ratio (NLR) at the Time of Transurethral Resection of Bladder Tumor: A Large Retrospective Study and Analysis of Racial Differences. Bladder Cancer. 2017;3(2):89-94.

8. Kawahara T, Furuya K, Nakamura M, Sakamaki K, Osaka K, Ito H, Ito Y, Izumi K, Ohtake S, Miyoshi Y, et al. Neutrophil-to-lymphocyte ratio is a prognostic marker in bladder cancer patients after radical cystectomy. BMC Cancer. 2016;16:185.

9. Rajwa P, Zyczkowski M, Paradysz A, Bujak K, Bryniarski P. Evaluation of the prognostic value of LMR, PLR, NLR, and dNLR in urothelial bladder cancer patients treated with radical cystectomy. Eur Rev Med Pharmacol Sci. 2018;22(10):3027-37.

10. Wang QH, Ji JL, Li H, He PL, Song LX, Zhao Y, Wang HY, Huang T, Sun XX, Cao YW, et al. [Preoperative Lymphocyte-to-monocyte Ratio Predicts Prognosis in Patients with Stage T1 Non-muscle Invasive Bladder Cancer]. Zhongguo Yi Xue Ke Xue Yuan Xue Bao. 2019;41(5):622-9.

11. Davalos D, Akassoglou K. Fibrinogen as a key regulator of inflammation in disease. Semin Immunopathol. 2012;34(1):43-62.

12. Zhu LR, Li J, Chen P, Jiang Q, Tang XP. Clinical significance of plasma fibrinogen and D-dimer in predicting the chemotherapy efficacy and prognosis for small cell lung cancer patients. Clin Transl Oncol. 2016;18(2):178-88.

13. Sanchez-Lara K, Turcott JG, Juarez E, Guevara P, Nunez-Valencia C, Onate-Ocana LF, Flores D, Arrieta O. Association of nutrition parameters including bioelectrical impedance and systemic inflammatory response with quality of life and prognosis in patients with advanced non-small-cell lung cancer: a prospective study. Nutr Cancer. 2012;64(4):526-34.

14. Wang X, Ni X, Tang G. Prognostic Role of Platelet-to-Lymphocyte Ratio in Patients With Bladder Cancer: A Meta-Analysis. Front Oncol. 2019;9:757.

15. Zhang L, Chen QG, Li SQ, Zhang J, Min QH, Gao QF, Sun F, Jiang YH, Wang XZ, Ying HQ. Preoperative fibrinogen to prealbumin ratio as a novel predictor for clinical outcome of hepatocellular carcinoma. Future Oncol. 2019;15(1):13-22.

16. Lv Y, Zhang J, Liu Z, Tian Y, Liu F. A novel inflammation-based prognostic index for patients with esophageal squamous cell carcinoma: Neutrophil lymphocyte ratio/prealbumin ratio. Med (Baltim). 2019;98(7):e14562.

17. Takeuchi H, Abe M, Takumi Y, Hashimoto T, Kobayashi R, Osoegawa A, Miyawaki M, Okamoto T, Sugio K. The prognostic impact of the platelet distribution width-to-platelet count ratio in patients with breast cancer. PLoS One. 2017;12(12):e0189166.

18. Sahni A, Francis CW. Vascular endothelial growth factor binds to fibrinogen and fibrin and stimulates endothelial cell proliferation. Blood. 2000;96(12):3772-8.

19. Sahni A, Khorana AA, Baggs RB, Peng H, Francis CW. FGF-2 binding to fibrin(ogen) is required for augmented angiogenesis. Blood. 2006;107(1):12631.

20. Gabay C. Interleukin-6 and chronic inflammation. Arthritis Res Ther. 2006;8(Suppl 2):3.

21. Ridker PM, Howard CP, Walter V, Everett B, Libby P, Hensen J, Thuren T, Group CPI. Effects of interleukin-1beta inhibition with canakinumab on hemoglobin A1c, lipids, C-reactive protein, interleukin-6, and fibrinogen: a phase Ilb randomized, placebo-controlled trial. Circulation. 2012;126(23):2739-48.

22. Bindea G, Mlecnik B, Fridman WH, Pages F, Galon J. Natural immunity to cancer in humans. Curr Opin Immunol. 2010;22(2):215-22.

23. Mantovani A, Allavena P, Sica A, Balkwill F. Cancer-related inflammation. Nature. 2008;454(7203):436-44.

24. Hanahan D, Weinberg RA. Hallmarks of cancer: the next generation. Cell. 2011;144(5):646-74.

25. Lee JL, Oh ES, Lee RW, Finucane TE: Serum Albumin and Prealbumin in Calorically Restricted, Nondiseased Individuals: A Systematic Review. Am J Med 2015, 128(9):1023 e1021-1022.

Page $9 / 19$ 
26. Liu X, Sun X, Liu J, Kong P, Chen S, Zhan Y, Xu D. Preoperative C-Reactive Protein/Albumin Ratio Predicts Prognosis of Patients after Curative Resection for Gastric Cancer. Transl Oncol. 2015;8(4):339-45.

27. Miura K, Hamanaka K, Koizumi T, Kitaguchi Y, Terada Y, Nakamura D, Kumeda H, Agatsuma H, Hyogotani A, Kawakami S, et al. Clinical significance of preoperative serum albumin level for prognosis in surgically resected patients with non-small cell lung cancer: Comparative study of normal lung, emphysema, and pulmonary fibrosis. Lung Cancer. 2017;111:88-95.

28. Measurement of visceral protein status in assessing protein and energy malnutrition: standard of care. Prealbumin in Nutritional Care Consensus Group. Nutrition 1995, 11(2):169-171.

29. Beck FK, Rosenthal TC. Prealbumin: a marker for nutritional evaluation. Am Fam Physician. 2002;65(8):1575-8.

30. Moghazy AM, Adly OA, Abbas AH, Moati TA, Ali OS, Mohamed BA. Assessment of the relation between prealbumin serum level and healing of skingrafted burn wounds. Burns. 2010;36(4):495-500.

31. Jagoe RT, Goodship TH, Gibson GJ. Nutritional status of patients undergoing lung cancer operations. Ann Thorac Surg. 2001;71(3):929-35.

32. Miyazono K, Yuki K, Takaku F, Wernstedt C, Kanzaki T, Olofsson A, Hellman U, Heldin CH. Latent forms of TGF-beta: structure and biology. Ann N Y Acad Sci. 1990;593:51-8.

33. Meikle CK, Kelly CA, Garg P, Wuescher LM, Ali RA, Worth RG. Cancer and Thrombosis: The Platelet Perspective. Front Cell Dev Biol. $2016 ; 4: 147$.

34. Li N, Kanda K, Fukumori T, Inoue Y, Nishitani M, Kanayama H, Kagawa S. Expression of vascular endothelial growth factor isoforms and plateletderived endothelial cell growth factor in bladder cancer. Urol Oncol. 2000;6(1):10-5.

35. Vagdatli E, Gounari E, Lazaridou E, Katsibourlia E, Tsikopoulou F, Labrianou I. Platelet distribution width: a simple, practical and specific marker of activation of coagulation. Hippokratia. 2010;14(1):28-32.

36. Paulus JM. Recent advances in the story of megakaryocyte physiology. Pathol Biol (Paris). 1981;29(3):133-5.

37. Matsuo K, Tang SH, Sharifi B, Rubin SA, Schreck R, Fagin JA. Growth factor production by human thyroid carcinoma cells: abundant expression of a platelet-derived growth factor-B-like protein by a human papillary carcinoma cell line. J Clin Endocrinol Metab. 1993;77(4):996-1004.

38. Sica A, Allavena P, Mantovani A. Cancer related inflammation: the macrophage connection. Cancer Lett. 2008;267(2):204-15.

39. Kaushansky K. Growth factors and hematopoietic cell fate. A new feature: controversies in hematology. Blood. 1998;92(2):345-344.

\section{Figures}

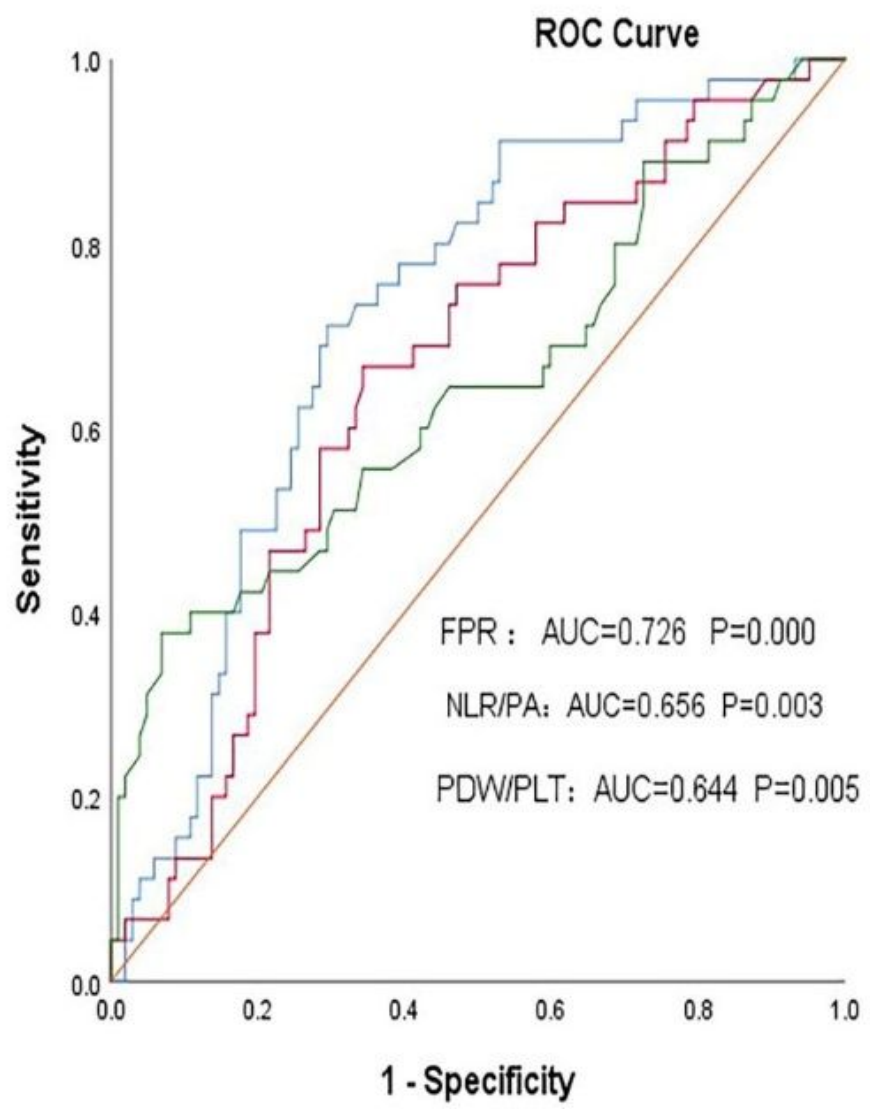

- FPR

- NLR/PA

-PDW/PLT

- Reference line

Figure 1

Page 10/19 
Relationship between FPR, NLR/PA, PDW/PLT and tumor progression

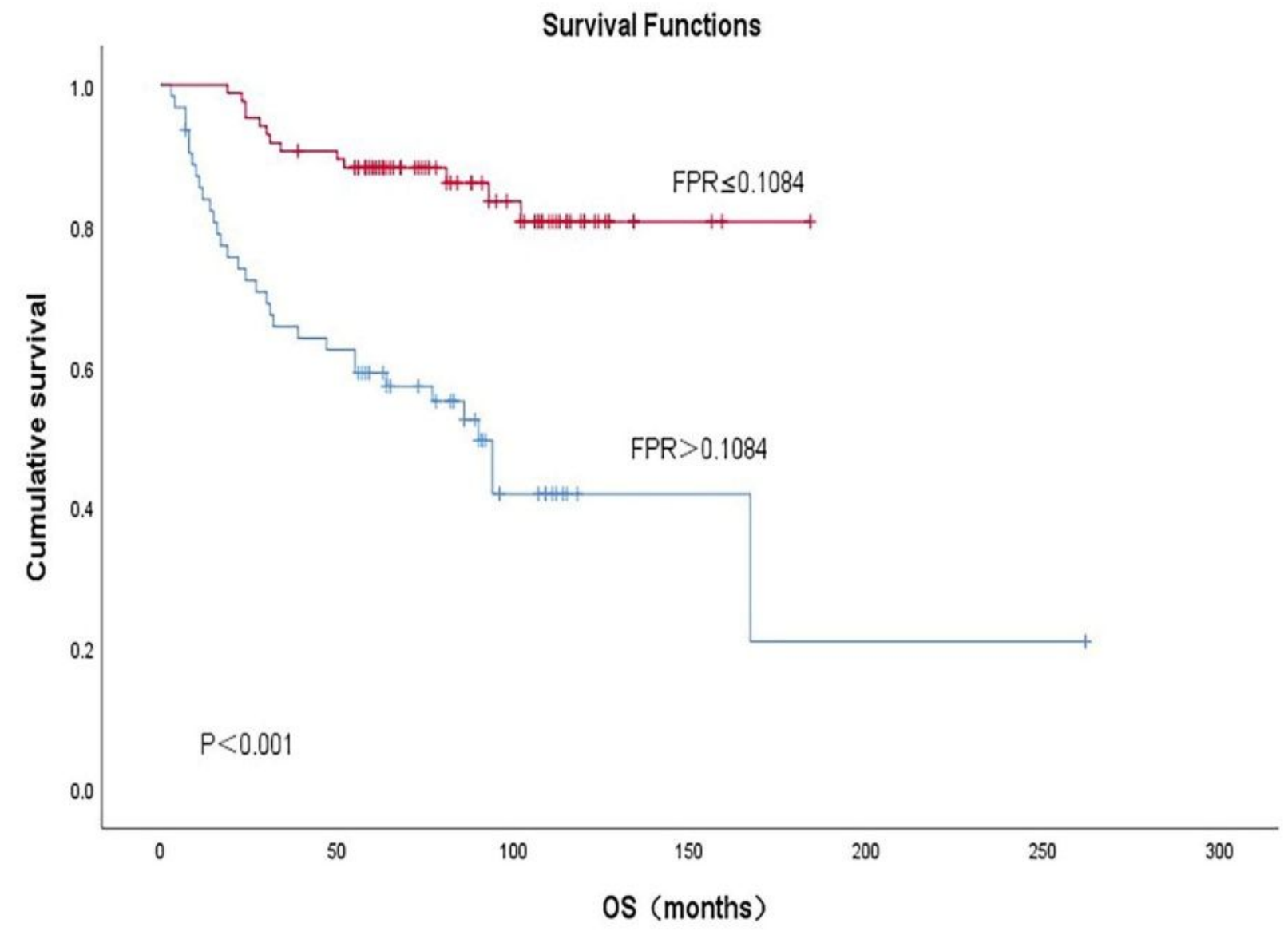

Figure 2

Comparison of OS between the two groups 


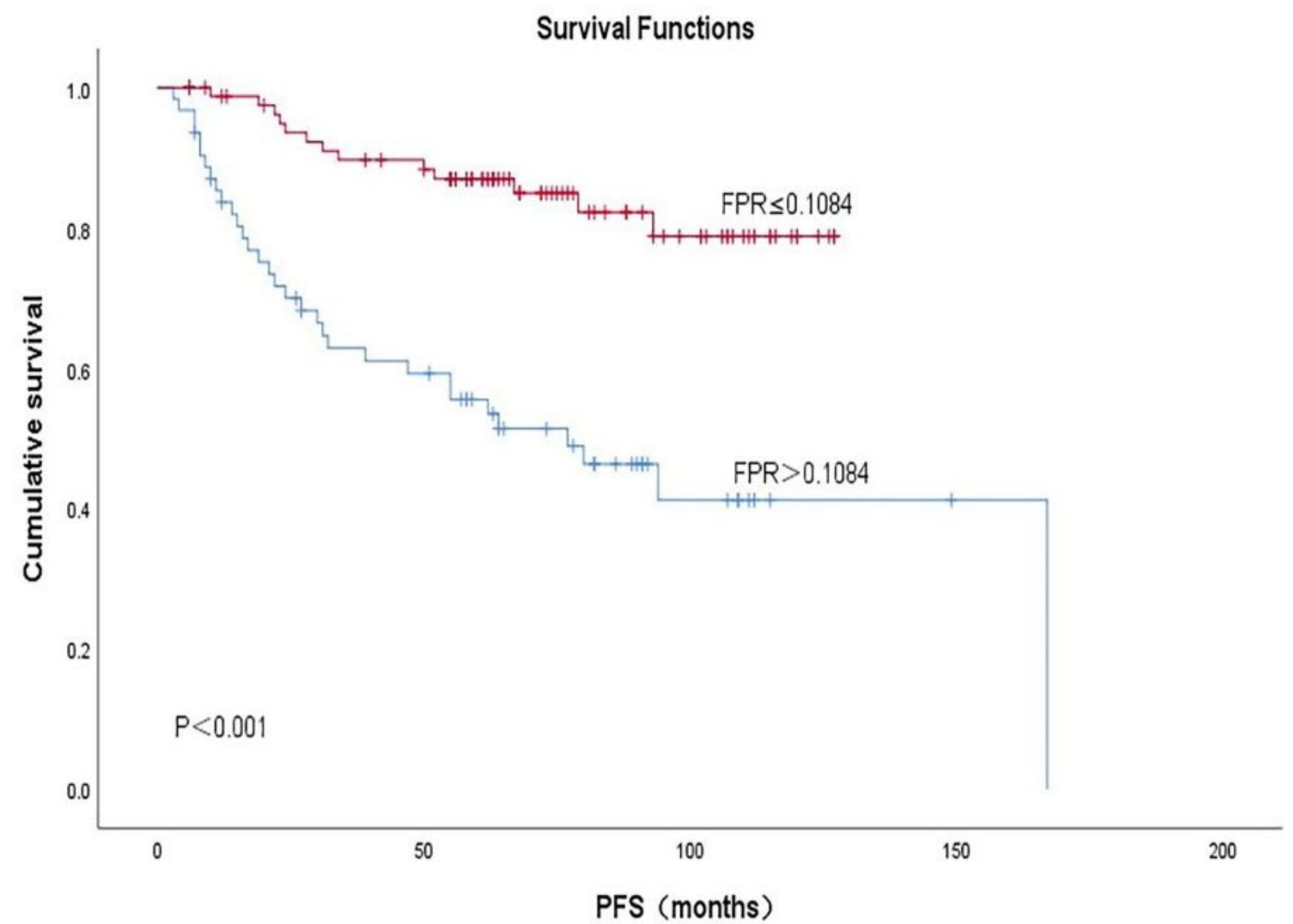

Figure 3

Comparison of PFS between the two groups 


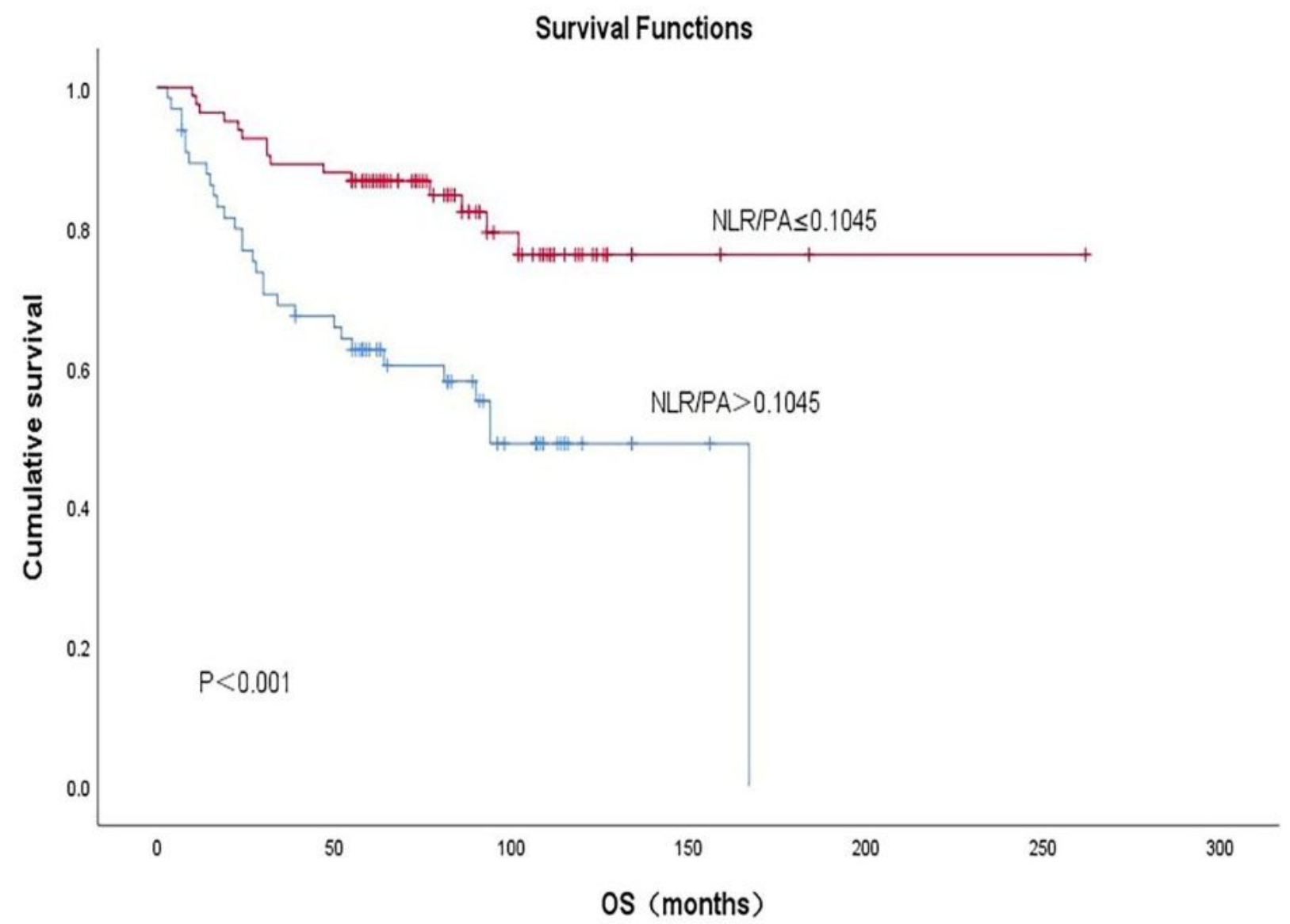

Figure 4

Comparison of OS between the two groups 


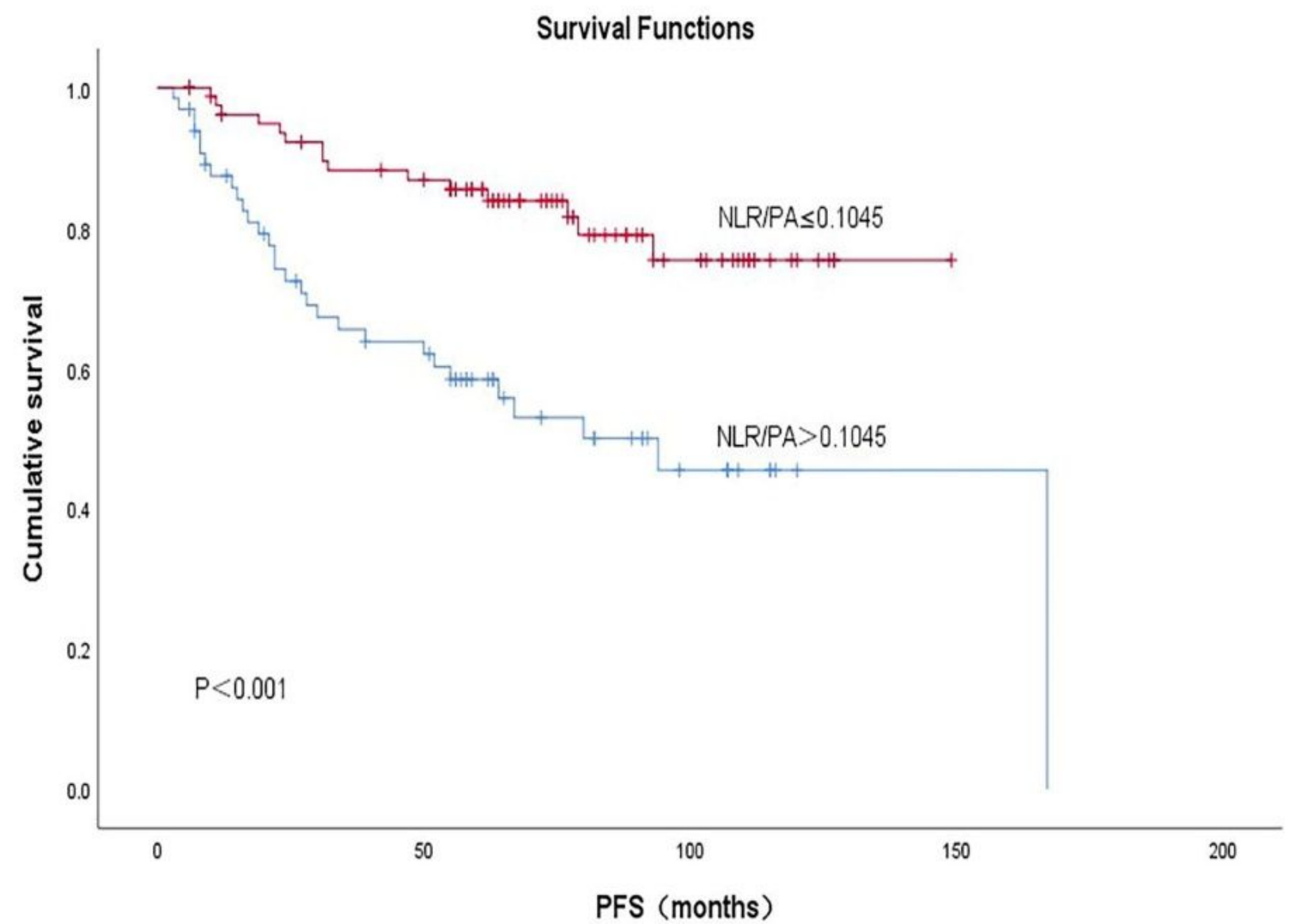

Figure 5

Comparison of PFS between the two groups 


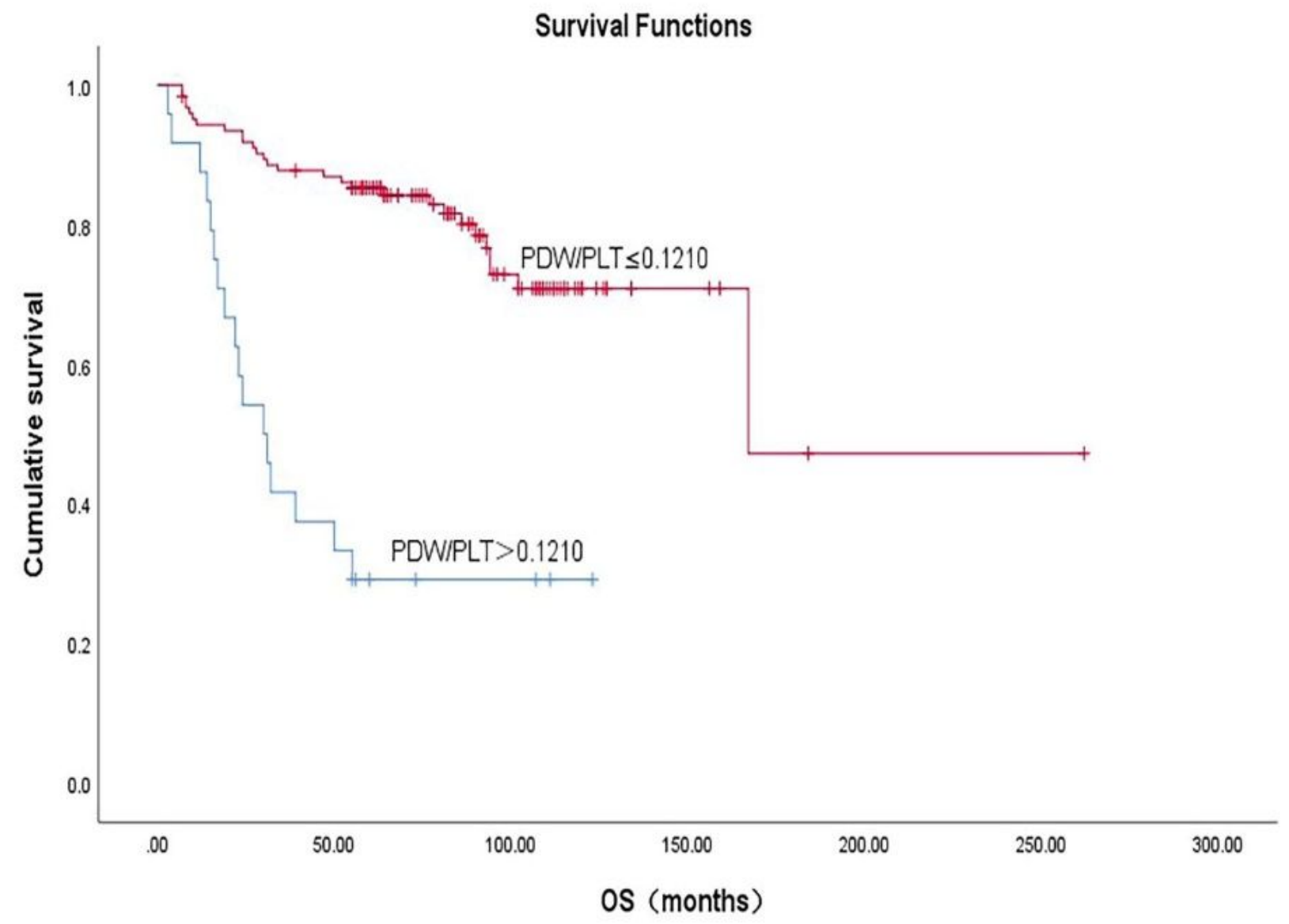

Figure 6

Comparison of OS between the two groups 


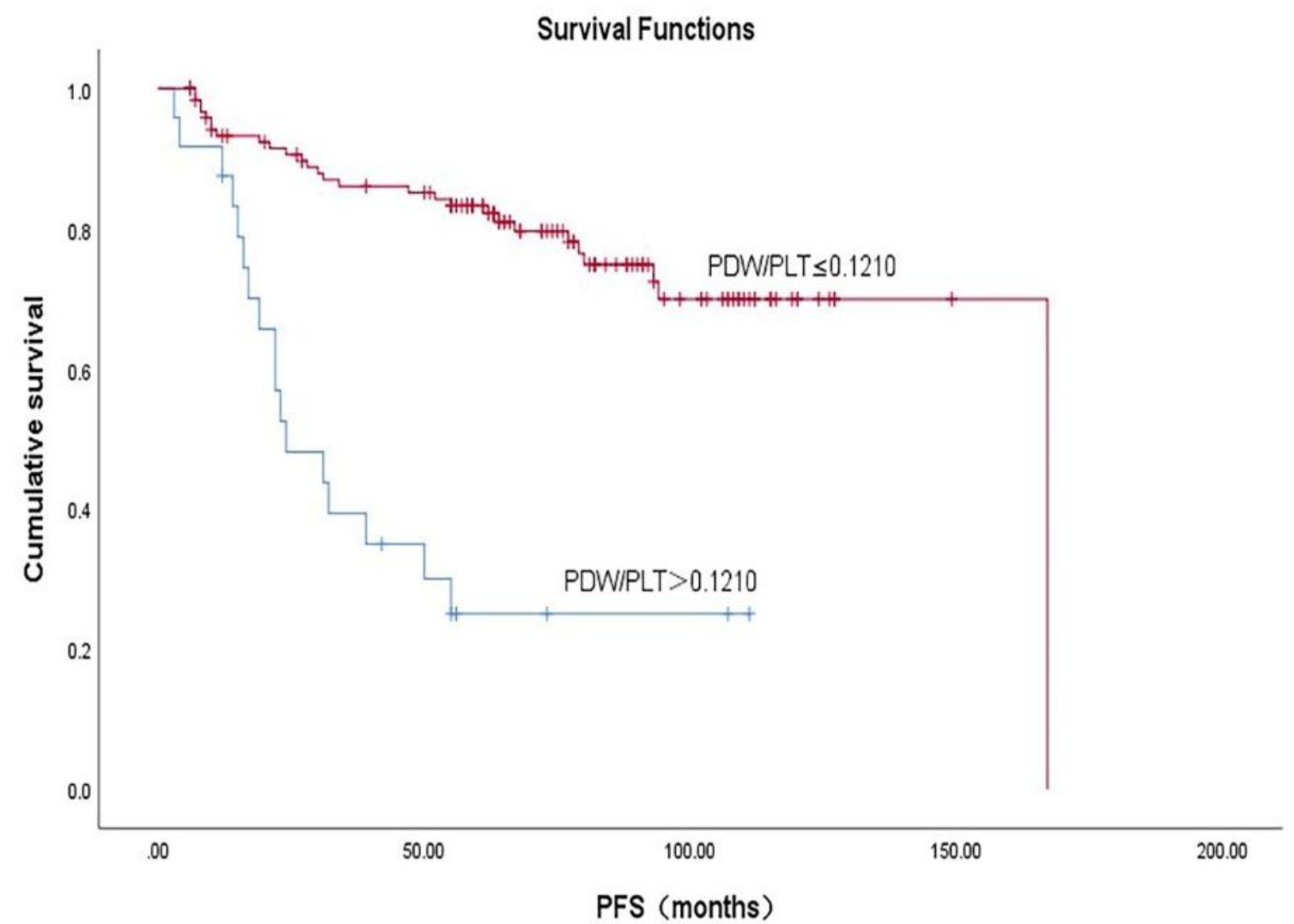

Figure 7

Comparison of PFS between the two groups 


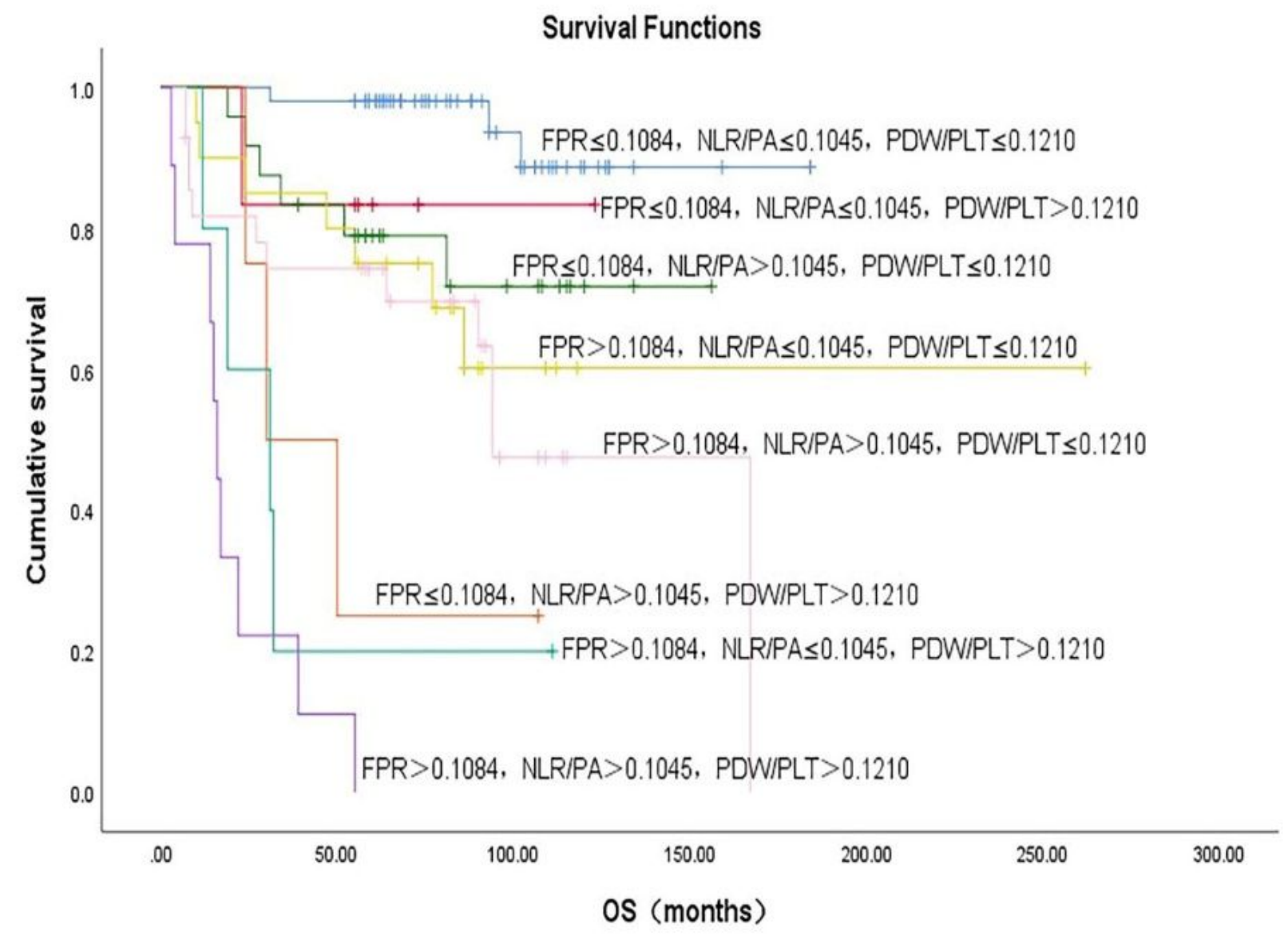

Figure 8

Comparison of OS of eight groups after stratified analysis 


\section{Survival Functions}

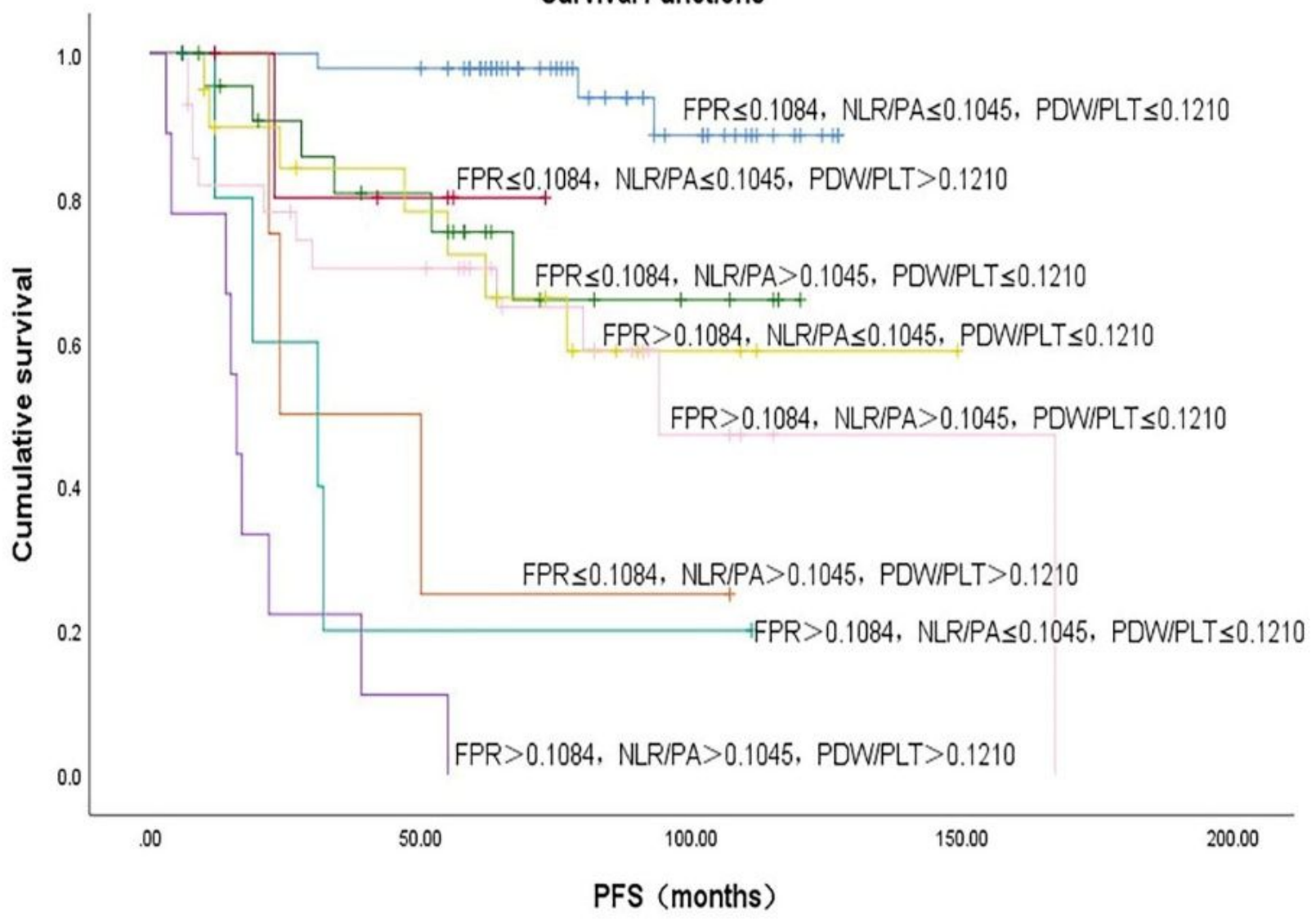

Figure 9

Comparison of PFS of eight groups after stratified analysis

Points

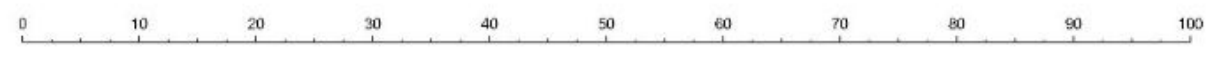

grade

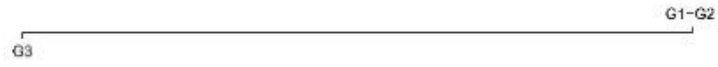

FPR

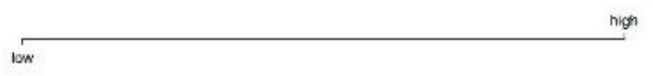

NLRJPA

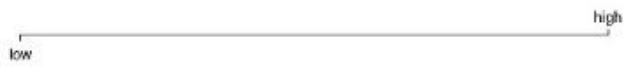

PDWPLT

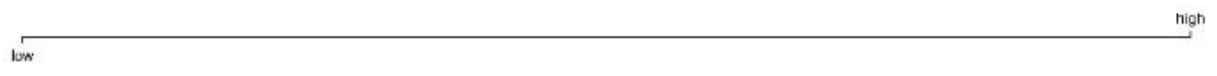

Total Points

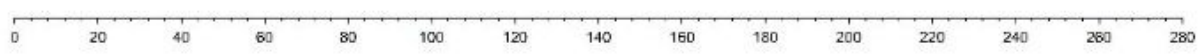

3-Year Survival

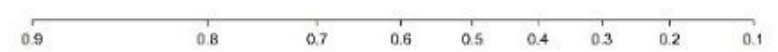

5-Year survival

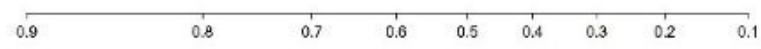


Figure 10

$\mathrm{R}$ language to draw nomogram and build prediction model

Page 19/19 